Pages: 1-13

http://irojournals.com/jscp/

DOI: https://doi.org/10.36548/jscp.2019.1.001

\title{
HYBRIDIZED GENETIC-SIMULATED ANNEALING ALGORITHM FOR PERFORMANCE OPTIMIZATION IN WIRELESS ADHOC NETWORK
}

\author{
Dr. Jennifer S. Raj, \\ Professor, \\ Department of Electronics \& Communication Engineering, \\ Gnanamani College of Technology, \\ Namakal, India. \\ Email: jennifer.raj@gmail.com \\ Dr. K. Rahimunnisa, \\ Associate Professor, \\ Department of ECE, \\ Easwari Engineering College, \\ Chennai. \\ Email: krahimunnisa@gmail.com
}

\begin{abstract}
The wireless Adhoc network are framed instantaneously utilizing the neighboring available mobile nodes and are capable of reconfiguring and healing. This autonomous behavior of the WANET and their other unique characteristics such as high mobility, limited battery power and dynamic topology changes incorporates several challenges in routing process for the transmission of the information. The traditional methods did not shows improvement and the existing methods also resulted with moderate improvements in performance of the WANET. So the paper proposes a hybridized method for the routing process combining the multi-populated genetic with simulated annealing to identify the shortest path and the fuzzy logic in detection of the malicious activities enhancing the performance of the network preventing the packet losses. The proposed method is validated using the network simulator-2 and its performance is analyzed.
\end{abstract}

Keywords: Wireless Adhoc network, Multi-populated genetic algorithm, Simulated annealing, Shortest path, Fuzzy classifier, malicious activity.

\section{Introduction}

The progressive growth in the wireless communication has paved way for the emergence of the more and more network that are mobile for e.g.: the mobile adhoc networks [8], the mobile adhoc networks or the wireless adhoc networks are the group of mobile nodes (data points) that behave autonomously, framing a adhoc network (designed specifically for a particular purpose) in a decentralized manner [9], the nodes involved may be homogenous or

ISSN: 2582-2640 (online)

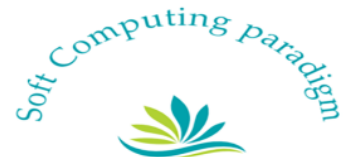


Journal of Soft Computing Paradigm (JSCP) (2019)

Vol.01/ No. 01

Pages: 1-13

http://irojournals.com/jscp/

DOI: https://doi.org/10.36548/jscp.2019.1.001

heterogeneous and the adhoc network framed are capable of self- organizing, reconfiguring and self-healing. The fig. 1 below shows the framing of the wireless adhoc network.

They form a network based on the availability of the device nearby that are ready to connect with them and engage as an individual network or a part of a paradigm that is larger. Despites its credentials such as self- healing, organizing and configuring the, mobile adhoc faces numerous of challenges that due to its characteristics. Some of them are dynamic topology changes, bandwidth constraints, energy constraints, varying link capacity, sovereign behavior and limited security. These above mentioned characteristics make the routing in the mobile or wireless adhoc network becomes difficult. So many research's where presented for handling the routing process of the MANET, to show efficiency in terms of the battery power, network lifetime, bandwidth utilization, congestion and cost, , most of the researcher's improvised the concept of the ADOV routing and the DS Routing by employing the intelligent algorithm's [9],[12] and soft computing methods [7],[10],[11] in turn to enhance the performance of the routing on the grounds of the delay, throughput, packet losses or drops, reliability, packet delivery ratio , and network lifetime.

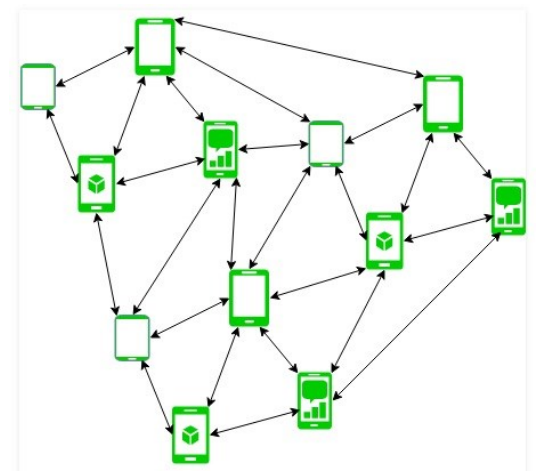

Fig .1 Wireless Adhoc Network [16]

The methods showed some improvement in the performance of the WANET with regard to few parameters at the cost of the other. The evolutionary algorithms and the other methods separately applied for the enhancing the quality of service of the MANET ended up only with limited improvements

So the paper proposes a combined algorithm that incorporates the multi-populated genetic algorithm with the simulated annealing for deciding with the optimal shortest route along with the energy conservation for the transmission of the information and the fuzzy logic in detecting and classifying of the malicious activities [14] to elude the packet losses during the transmission and improve the throughput.

ISSN: 2582-2640 (online)

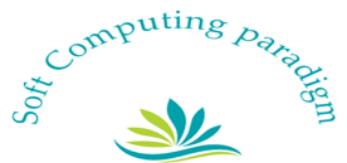


Journal of Soft Computing Paradigm (JSCP) (2019)

Vol.01/ No. 01

Pages: $1-13$

http://irojournals.com/jscp/

DOI: https://doi.org/10.36548/jscp.2019.1.001

The remaining paper is organized with the 2. Related works giving the routing techniques developed for MANET utilizing various intelligent algorithms and soft computing techniques. 3. The proposed work of identifying the shortest path applying the multi-populated Genetic- simulated annealing and the fuzzy in the detection of the malicious activities. 4. Provides the performance analysis of the hybridized process and the other methods. 5. Conclusion.

\section{Related Works}

In order to improvise the convergence speed and have global optimum the traditional genetic algorithm that was surveyed by the Srinivas et al [2], presenting the detailed study and the analogy of the genetic algorithm despites its advantages of finding the fittest solution, the traditional GA resulted with the early convergence so the genetic algorithm was improvised by developing hybridized method. Li et al [1] proposed a hybridized genetic algorithm aiming at an enhanced convergence to arrive at an optimal solution. As the paper concentrates on the developing the performance optimization method for the wireless adhoc network. Conti, et al [4] providing a detailed survey of the mobile adhoc network and the network structures emerged from it, and its incompatibility with the computer communications. Pathan et al [3] the survey on the security of the self-organizing networks, mobile adhoc networks, wireless sensor network, wireless mesh network, vehicular network was done in the paper. Finding an able routing for the wireless adhoc network was the complex and a time consuming task. Bheemalingaiah et al [5] proposed an energy aware routing based on the disjoint multipath routing in the mobile adhoc network. The routing step for the wireless adhoc network concentrated on the reducing the energy consumption and the improving the network longevity, more methods with the researches of enhancing the networking longevity and the quality of the routing was proposed. Tilwari, et al [6] proposed one such routing with the addressing the technical constraint of the mobile adhoc network, such the mobility, residual energy, link quality and the battery availability employing the q-learning results with the overloaded states that reduce the results, and end up with a minimum behavior. Then the fuzzy logic based clustering of Nayak, et al [7] concentrated in improving the performance of the wireless sensor network that is the subtype of wireless adhoc network. Using the fuzzy rule based system with the system trained using the remaining battery, mobility of the base station, and the location of the nodes. Yang et al [8], the randomly changing topology of the mobile adhoc networks are handled by employing a memory and immigrant based GA that is compatible with the changing network topology Robinson et al [9], to further enhance the performance of the WANET the author proffers a PSO-RNN model to find an loop free path that is optimal to for the transmission to be cost-effective, with the optimized energy and traffic ratio. Kumar et al [10], the paper proposes a neural network based congestion control technique to have a reliable transmission, with the capability of recognizing and capturing the mobility behavior. Xie, et al [11], employs the ADOV routing with the fuzzy neural network and simulated

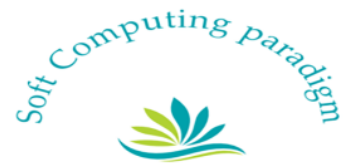


Journal of Soft Computing Paradigm (JSCP) (2019)

Vol.01/ No. 01

Pages: 1-13

http://irojournals.com/jscp/

DOI: https://doi.org/10.36548/jscp.2019.1.001

annealing with the genetic algorithm for the vehicular adhoc networks to find out the malicious activities that are to happen in the path by enabling the trust evaluation for the nodes Labed et al [12], the paper proposes the AODV routing protocols along with the bee colony optimization to address the issues of routing in the MANET. Anand et al [13] the packet loses or the drops during the transmission is reduced by employing an Adhoc on- demand routing to optimizing the batter power so as to reduce the packet drops and the losses Bisen et al [14] the paper proposes a fizzy based classifier in identifying the malicious activities in order to reduce the packet losses incurred during the transmission of the packets. Lanjewar, et al [15] the paper proposes a check point method for the MANET by applying the reinforcement learning enable a self-organizing routing for the MANET.

\section{Proposed Work}

The wireless adhoc network facing multitudes of issues, due to the characteristics it possess, incurs many challenges in the process of routing. Some of the challenges where energy consumption, mobility, low power availability, that all resulted with the loss in the network lifetime, causing failures in the transmission of the packets. So the paper proposes a combination of intelligent algorithms that is the multi-populated genetic algorithm with the simulated annealing to identify the shortest path and employ the fuzzy logic in identifying the malicious activities in the nodes [14]. So as to show enhancement in the performance of the wireless adhoc networks, in terms of throughput, packet delivery ratio and the accuracy of the detection.

\subsection{Problem Definition}

The problem of the finding the shortest path for the MANET considering the energy availability $\left(E_{a}\right)$, the mobility $\left(M_{n}\right)$, and the distance of the nodes from the destination $\left(D_{n}\right)$ is formulated as the undirected graph $G(V, E)$ where, the $V=\left\{n_{1}, n_{2}, \ldots \ldots . n_{i}\right\}$ represents the number of nodes and the and the $E$ represents the objectives functions to be optimized in order to improve the performance of the network applying the multi-populated genetic with simulated annealing. The multi-populated genetic with simulated annealing is applied to identify the fittest nodes with the maximum of $E_{a}$ minimum of $M_{n}$ and $D_{n}$, in order to estimate the shortest path and further uses the fuzzy logic, classification and the detection [14] to identify the malicious activities in the nodes.

So the proposed method is proceeded as two stages.

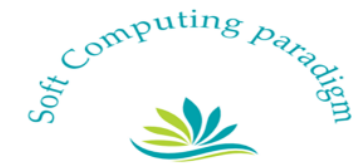


Journal of Soft Computing Paradigm (JSCP) (2019)

Vol.01/ No. 01

Pages: $1-13$

http://irojournals.com/jscp/

DOI: https://doi.org/10.36548/jscp.2019.1.001

- Identifying the shortest path by enumerating the nodes with $\operatorname{MAX}\left(E_{a}\right), \operatorname{MIN}\left(M_{n}\right)$ and $\operatorname{MIN}\left(D_{n}\right)$

- Reducing the malicious attacks by classifying the malicious activities using the fuzzy classifier

\subsection{Multi-Populated Genetic Algorithm}

The multi-populated genetic algorithm splits the entire population into sub-population and applies the process of standard genetic algorithm that includes the selection, and evaluates for the fittest individual, if identified stops with the elite individual selected and replaces it with the worst individual in the next population or used to initiate the next population. It the criteria not satisfied the process is extended with the crossover and the mutation and fitness evaluation to identify the elite individual in the population to replace the best individual with the worst individual and converges with a global optimum solution. This improves the speed of the process compared to the single population algorithm.

\subsection{Simulated Annealing}

Process of simulated annealing is used to attain a global optimum by identifying the local optimum. The procedure of the simulated annealing is overlaid on the concepts of thermodynamics, as it includes the temperature in the steps of minimization. The high temperature is utilized in exploring the parameter space and the low temperature stops the exploration of the parameter space. For each temperature the repeats with the steps of initiating a population, evaluating individuals and accepting the individuals that improve and do not improve the solution and arrives at a global optimum if the cooling of the temperature is sufficiently slow. So the probability of the acceptance depends on the temperature.

\subsection{Proposed Method of Shortest Path Identification using the Multi-populated GA with SA (M-PGSA)}

The shortest path routing problem of the WANET is handled in the paper by applying the combined multi-populated GA with simulated annealing to arrive at a global solutions. To have a shortest path transmission and a continuous transmission, the nodes with the $\operatorname{MIN}\left(D_{n}\right)$ from the destination are gathered along with the nodes with the $\operatorname{MAX}\left(E_{a}\right)$, and the $\operatorname{MIN}\left(M_{n}\right)$. The proposed method of multi-populated GA with the SA is applied to identify the nodes holding all the three attributes. The nodes enriched with all the three attributes are identified in order to extend a continuous and a short path between the sources to the destination. The request (RQST) from the

ISSN: 2582-2640 (online)

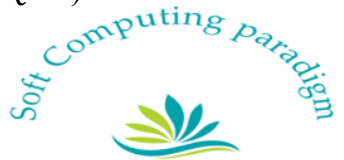


Journal of Soft Computing Paradigm (JSCP) (2019)

Vol.01/ No. 01

Pages: $1-13$

http://irojournals.com/jscp/

DOI: https://doi.org/10.36548/jscp.2019.1.001

destination is broadcasted to all the neighboring nodes to identifying the interested nodes taking part in the network. The request transmitted for the identification of the willing neighbor nodes also asks for the attributes of the neighboring nodes related to the mobility, distance from the destination and the energy availability of the nodes. The willing nodes retransmits a reply $(R P L Y)$ with the information's requested for. Now the nodes gathered are enumerated applying the M-PGSA algorithm to identify the befitting nodes taking part in the routing process for having a continuous transmission and for enumerating the shortest path.

The steps involved in the identification of the befitting nodes and the shortest path are as follows. Identification of befitting nodes

Step 1: Initialize population with the nodes $\left\{n_{1}, n_{2}, \ldots \ldots n_{i}\right\}$ willing to take part in the transmission.

Step2: initialize the population size, counter for the evolution with $E_{V}=0$, and the temperature $\operatorname{Tm} p=T$

Step 3: Evaluate the nodes extracting the information's gathered on the $E_{a}, M_{n}$ and $D_{n}$

Step 4: IF befitting node obtained, replaces the worst node with the best node obtained and enumerates the next.

Step 5: ELSE performs the Crossover, Mutation and the Fitness evaluation.

Step 6: Continue with the next node, if identified with the best.

Step 7: Further SA is applied to estimate whether the solution obtained is a global optimum.

Step 8: Enumerates Fitness

Step 9: Terminates IF satisfied Else continues, obtains the best global solution and terminates

(ii) Enumerating the Shortest path

The befitting nodes identified are added to the table that constitutes the neighboring node information and once again enumerated to identify the shortest path. The M-PGSA is further applied for the enumeration of the shortest path that is fittest, gathering the nodes with the $M I N\left(D_{n}\right)$ from the destination. For e.g. the destination broadcasting the request message for the include the path with the nodes that have a very minimum distance to the destination than the intermediate forwarding node and drops the other. The M-PGSA applied enumerates for such fittest shortest path and updates to the table contained with the routing information. So each time the source with the urge to transmit information, selects the fittest, shortest path available in the table without any delay, and energy consumption on discovering the route for the process of the transmission. The path enriched with the befitting nodes enables a continuous transmission also. Though the path is enriched with the nodes of the maximum energy, minimum distance and minimum mobility. The path are likely to be affected by the malicious attacks causing losses

ISSN: 2582-2640 (online)

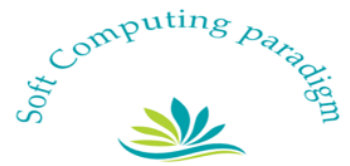


Journal of Soft Computing Paradigm (JSCP) (2019)

Vol.01/ No. 01

Pages: $1-13$

http://irojournals.com/jscp/

DOI: https://doi.org/10.36548/jscp.2019.1.001

of the packets. So there is a necessity to classify the nodes malicious attacks from the nodes enumerated with the $\operatorname{MAX}\left(E_{a}\right), M I N\left(M_{n}\right)$ and $M I N\left(D_{n}\right)$.So the proposed method includes a fuzzy classifier in identifying the path with the malicious attacks to enhance the performance by reducing the packet losses.

\subsection{Fuzzy Classifier in Identifying the Malicious Attack paths}

The process of identifying the malicious activity in the path established is done by the fuzzy classifier. After the commencement of the each individual transmission. The information regarding the delivery ratio of the packets $\left(P_{D R}\right)$, the amount of the packets sent $\left(P_{S}\right)$ and the amount of packet received $\left(P_{r}\right)$ are collected along with the $E_{a}$, and $M_{n}$ are monitored. The values extracted from the information's monitored are converted into a normalized value $\left(N R M_{v a l}\right)$ as either HIGH or LOW using the following equations (1)

$$
N R M_{\text {val }}=\left\{\begin{array}{l}
\frac{P_{D R}}{\operatorname{MAX}\left(P_{D R}\right)} \text { for noramlized } P_{D R} \\
\frac{P_{S}, P_{r}}{\operatorname{MAX}\left(P_{S}, P_{r}\right)} \text { for normalized } P_{S}, P_{r} \\
\frac{E_{a}}{\operatorname{MAX}\left(E_{a}\right)} \text { for mnormalized } E_{a} \\
\frac{M_{n}}{\operatorname{MAX}\left(M_{n}\right)} \text { for normalized } M_{n}
\end{array}\right.
$$

The information monitored and the normalized values obtained are utilized in training the network, with the capability of identifying the malicious activity in the path. The fuzzy employs the generalized bell shaped membership function in specifying the fuzzy sets (mapping the input values obtained to a normalized value 0 or 1 ). The equation (2) gives the fuzzy membership function.

$$
\mu_{x}(f)=\frac{1}{1+\left|\frac{f-c_{x}}{a_{x}}\right|^{\wedge} 2 b}
$$

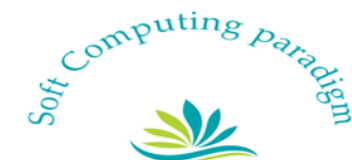


Journal of Soft Computing Paradigm (JSCP) (2019)

Vol.01/ No. 01

Pages: $1-13$

http://irojournals.com/jscp/

DOI: https://doi.org/10.36548/jscp.2019.1.001

The completion of the training proceeds with the testing phase in identifying the malicious activity in the path during the transmission. Network is trained with the node characteristics based on the $P_{D R}, P_{s}, P_{r}, E_{a}$ and $M_{n}$ and the regular training done ensures the detection of the malicious activities, the path identified with severe malicious activities is classified as the worst path and elude from the table. The fuzzy set obtained in the output are converted back into the de-normalized value by engaging the de-fuzzification step. The equation (3) is framed in this regard.

$$
\bar{f}=\int \mu_{x}(f) f d f / \int \mu(f) d f
$$

Where $\bar{f}$ is the de-fuzzification output and the $\mu(f)$ is the member ship and $f$ is the output. The flow chart in the fig. 1 explains the fuzzy interference in identifying the malicious activities.

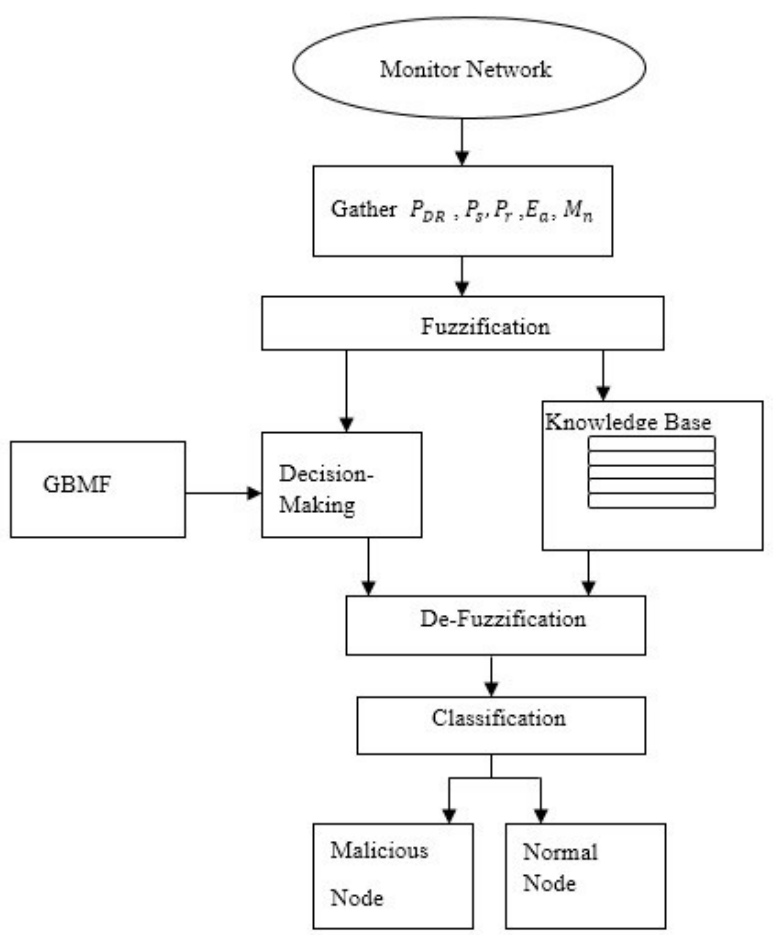

Fig .1 Fuzzy Rule Base

The flowchart gives the flow in the process of identifying the malicious activity in the path by identifying the nodes that pave way to the dropping of the packets result in the losses. The algorithm below in fig.2 details the proposed process of identifying the shortest path utilizing the M-PGSA and the malicious activity identification applying the fuzzy interference system.

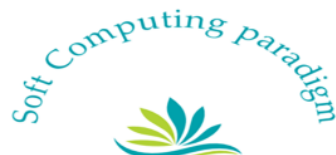


Journal of Soft Computing Paradigm (JSCP) (2019)

Vol.01/ No. 01

Pages: $1-13$

http://irojournals.com/jscp/

DOI: https://doi.org/10.36548/jscp.2019.1.001

The regular and the periodic training done, improves the capability of the network in identifying the unwanted threats that leads to the losses in the packets forwarded in turn resulting in decreased packet delivery ratio and the throughput, the proposed method enables the identification of the shortest path, reduces the energy in the process of transmission

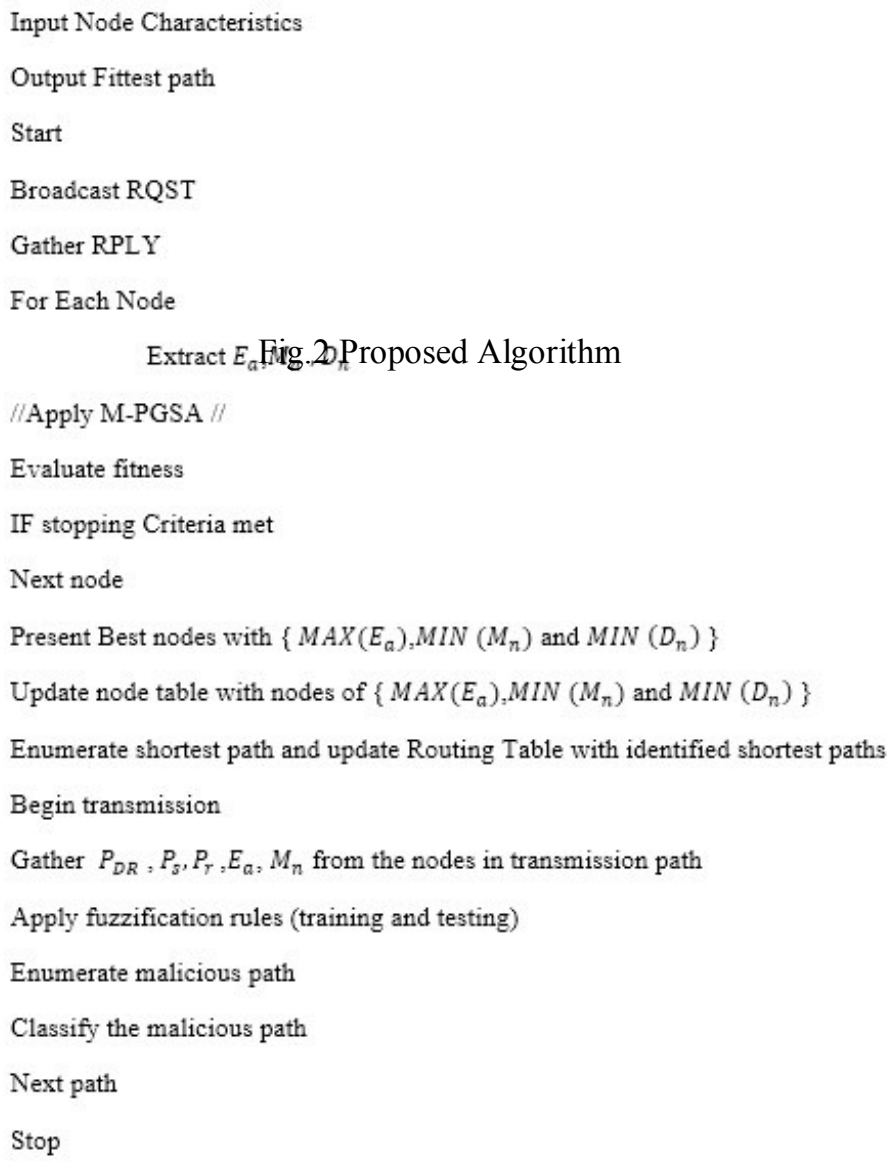

and improves the network performance by increasing the network lifetime. Further the fuzzy employed, identifies the unwanted threats and stops them by classifying the worst path. So the path enriched with trusted nodes with

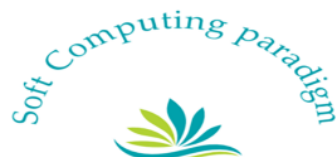


Journal of Soft Computing Paradigm (JSCP) (2019)

Vol.01/ No. 01

Pages: $1-13$

http://irojournals.com/jscp/

DOI: https://doi.org/10.36548/jscp.2019.1.001

maximum energy availability, minimum distance from the destination and minimum speed, provides with the improved performance for the wireless adhoc networks.

\section{Result Evaluation}

The proposed model is simulated using the network simulator-II, with the number of nodes varying from $100-500$ within an area of $1000 * 1000$ Sq.m, the simulation time of 100 seconds, initial energy of 100joules. The proposed method as wells the genetic algorithm, simulated annealing, the neural network and PSO are evaluated using the network simulator-II. The simulation results obtained proves the proposed method performs well compared to the other methods evaluated. The table.1 below gives the detail of the fuzzy rule base.

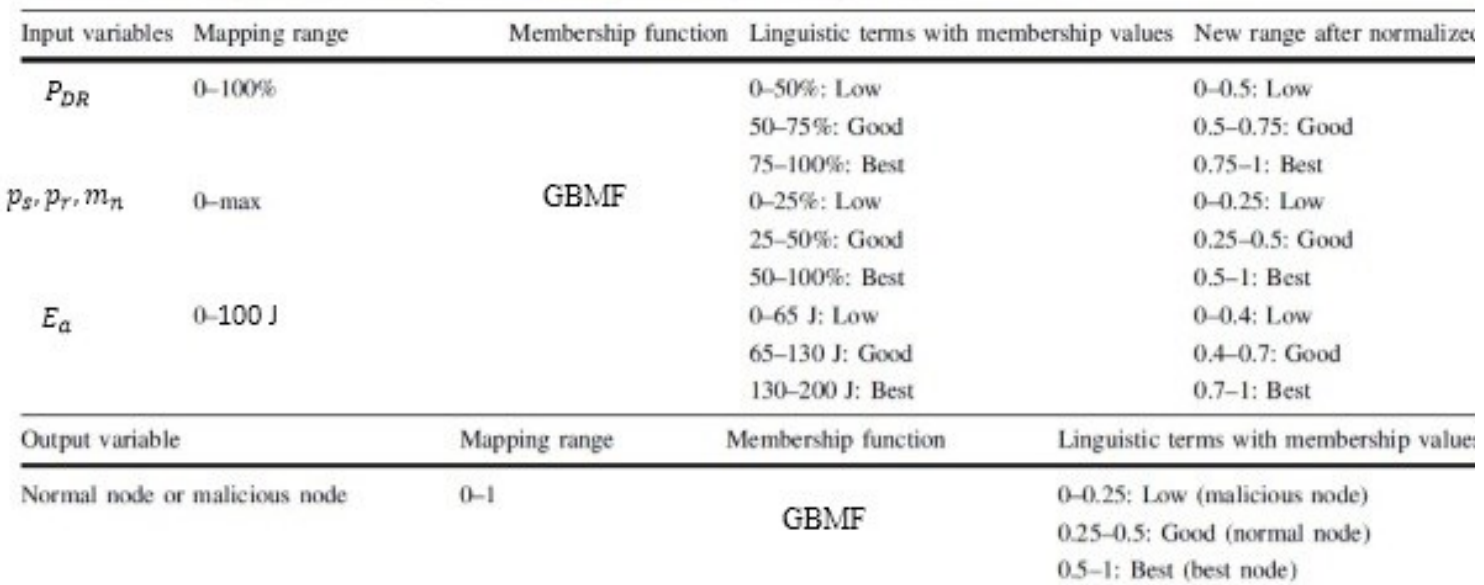

Table .1 Fuzzy knowledge Base [14]

The fig. 3 gives the simulation results obtained for the proposed method and the other methods genetic, particle swarm, simulated annealing, and neural network for varying number of nodes. The results obtained shows that the proposed method shows considerable improvements than the other methods that were evaluated.

ISSN: 258
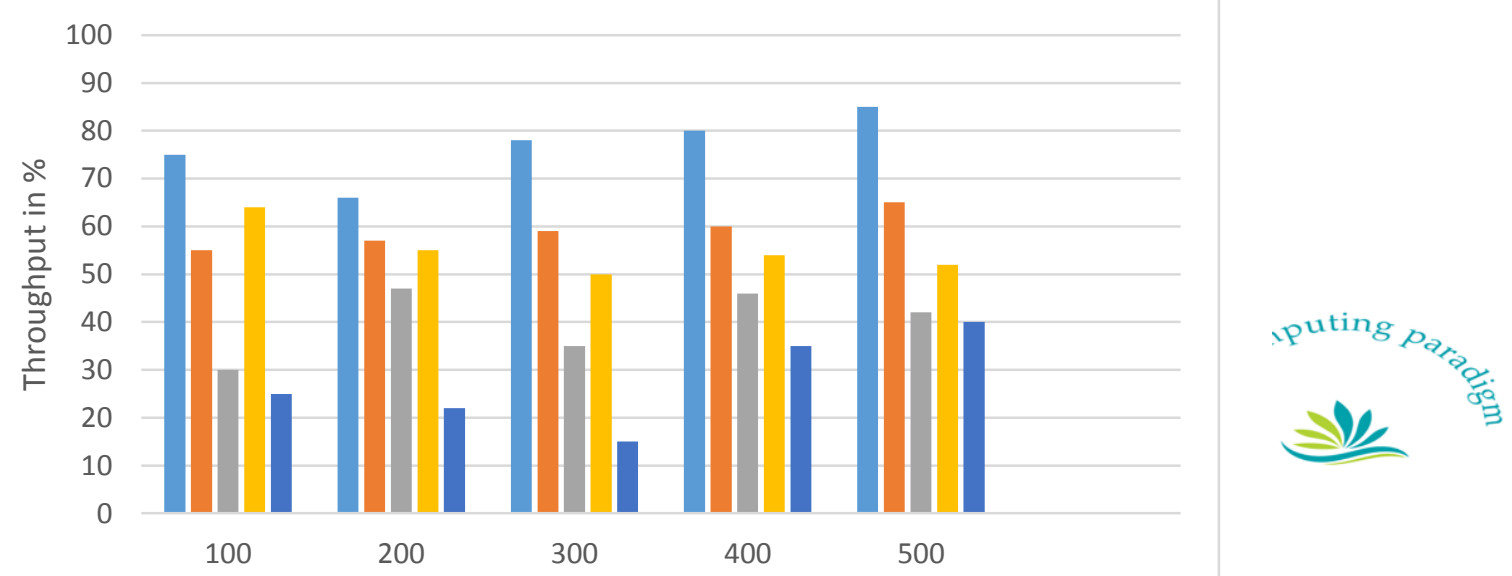
Journal of Soft Computing Paradigm (JSCP) (2019)

Vol.01/ No. 01

Pages: $1-13$

http://irojournals.com/jscp/

DOI: https://doi.org/10.36548/jscp.2019.1.001

The fig. 4 shows the simulation results for the packet delivery ratio of the proposed method and the other methods, genetic, particle swarm, simulated annealing, and neural network for number of nodes ranging from 100-500, the PDR of the proposed method is $30 \%$ improvised than the GA, 25\% improvised than the PSO, $20.57 \%$ improvised than the neural networks and $23.25 \%$ improvement than the simulated annealing.

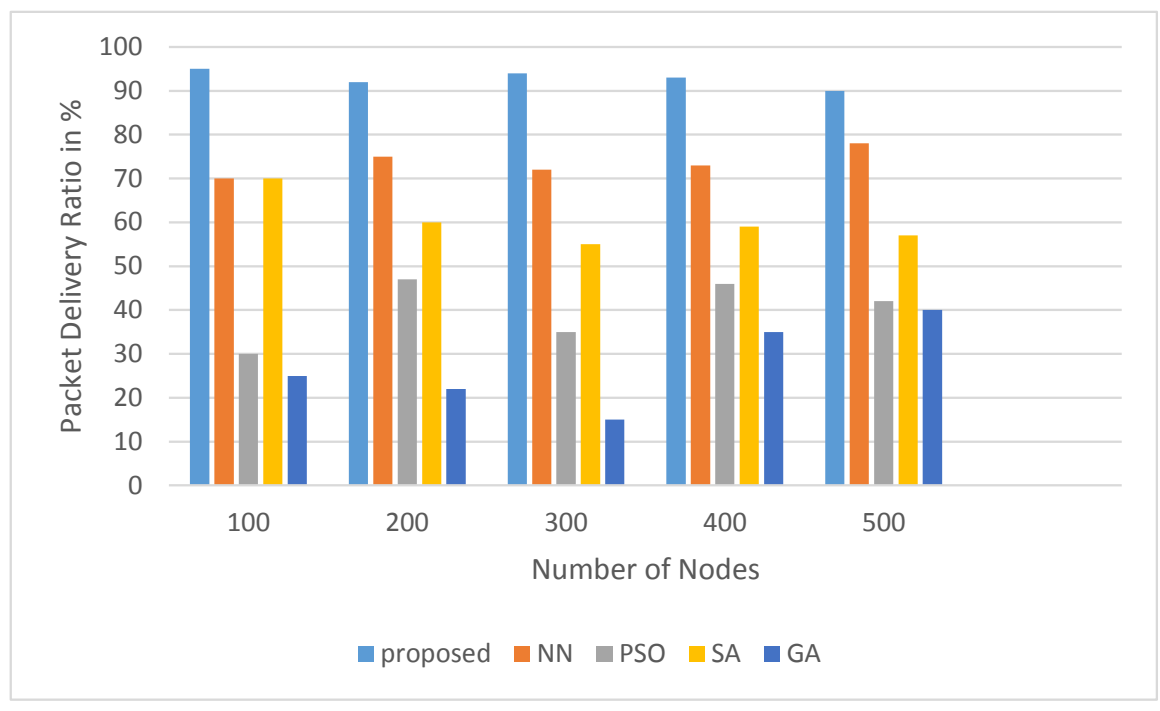

Fig .4 Packet Delivery Ratio

The fig. 5 gives the simulation results obtained on the lifetime of the network, for the proposed method and the other algorithms, genetic, PSO, neural network and simulated annealing. A considerable improvement is shown by the proposed method that improves the energy consumption of the network by the shortest path identification, more over

ISSN: $2582-2641$

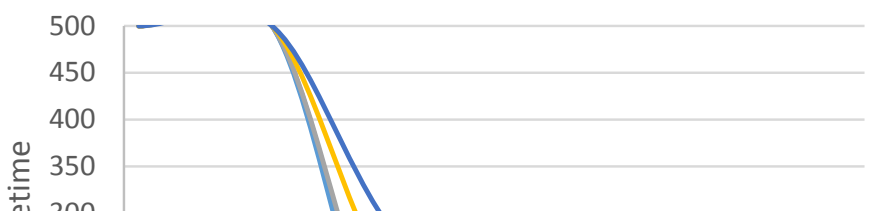


Journal of Soft Computing Paradigm (JSCP) (2019)

Vol.01/ No. 01

Pages: $1-13$

http://irojournals.com/jscp/

DOI: https://doi.org/10.36548/jscp.2019.1.001

the proactive way of routing enables to reduce the energy to a considerable amount thus extending the lifetime of the network.

Thus the simulation results obtained proves that the proposed hybridized method, improves the performance of the system to a certain level than the other methods. The path enriched with nodes containing maximum residual energy possessing a minimum distance to the destination and minimum mobility, reduce the energy consumption and extends a continuous transmission. Further the malicious activity detection and classification using the fuzzy rule base, enables the continuous transmission of the information without any hindrance.

\section{Conclusion}

The wireless adhoc network possessing unique characteristics that involves many challenges in developing a routing is handled in the paper. The paper proposes a hybridized method incorporating the genetic and the simulated annealing in identifying the shortest path for the process of transmission utilizing the information of the energy availability of the nodes, its distance and mobility. The shortest path enumerated reduces distance of the transmission in-turn reducing the energy consumption, increasing the network lifetime. The paper also incorporates the fuzzy classifier along with the proposed method to enumerate the unwanted access in the network and elude them. The performance evaluation of the proposed hybridized method and the other methods is done using the network simulator-2 to evince the improvements in the WANET through the proposed method than the other method.

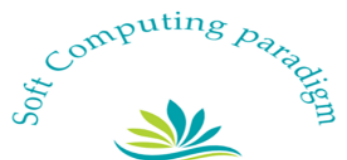


Journal of Soft Computing Paradigm (JSCP) (2019)

Vol.01/ No. 01

Pages: $1-13$

http://irojournals.com/jscp/

DOI: https://doi.org/10.36548/jscp.2019.1.001

\section{References}

[1] Li, Kun, Liwei Jia, and Xiaoming Shi. "An Efficient Hybridized Genetic Algorithm." In 2018 IEEE International Conference of Safety Produce Informatization (IICSPI), pp. 118-121. IEEE, 2018.

[2] Srinivas, Mandavilli, and Lalit M. Patnaik. "Genetic algorithms: A survey." computer 27, no. 6 (1994): 17-26.

[3] Pathan, Al-Sakib Khan, ed. Security of self-organizing networks: MANET, WSN, WMN, VANET. CRC press, 2016.

[4] Conti, Marco, and Silvia Giordano. "Mobile ad hoc networking: milestones, challenges, and new research directions." IEEE Communications Magazine 52, no. 1 (2014): 85-96.

[5] Bheemalingaiah, M., M. M. Naidu, D. Sreenivasa Rao, and G. Varaprasad. "ENERGY AWARE NODE DISJOINT MULTIPATH ROUTING IN MOBILE AD HOC NETWORK." Journal of Theoretical \& Applied Information Technology 5, no. 4 (2009).

[6] Tilwari, Valmik, Kaharudin Dimyati, M. H. D. Hindia, Anas Fattouh, and Iraj Sadegh Amiri. "Mobility, residual energy, and link quality aware multipath routing in MANETs with Q-learning algorithm." Applied Sciences 9, no. 8 (2019): 1582.

[7] Nayak, Padmalaya, and Anurag Devulapalli. "A fuzzy logic-based clustering algorithm for WSN to extend the network lifetime." IEEE sensors journal 16, no. 1 (2015): 137-144.

[8] Yang, Shengxiang, Hui Cheng, and Fang Wang. "Genetic algorithms with immigrants and memory schemes for dynamic shortest path routing problems in mobile ad hoc networks." IEEE Transactions on Systems, Man, and Cybernetics, Part C (Applications and Reviews) 40, no. 1 (2009): 52-63.

[9] Robinson, Y. Harold, and M. Rajaram. "Energy-aware multipath routing scheme based on particle swarm optimization in mobile ad hoc networks." The Scientific World Journal 2015 (2015).

[10]Kumar, Pravin, Sachin Tripathi, and Pankaj Pal. "Neural network based reliable transport layer protocol for MANET." In 2018 4th International Conference on Recent Advances in Information Technology (RAIT), pp. 1-6. IEEE, 2018.

[11] Xie, Tongyi, Jiawei Mo, and Baohua Huang. "A Secure AODV Protocol Improvement Scheme Based on Fuzzy Neural Network." In International Conference on Security and Privacy in Communication Systems, pp. 453-467. Springer, Cham, 2018.

[12] Labed, Said, Akram Kout, and Salim Chikhi. "A New Approach based Bee Colony for the Resolution of Routing Problem in Mobile Ad-Hoc Networks." International Journal of Applied Metaheuristic Computing (IJAMC) 10, no. 2 (2019): 131-151.

[13] Anand, M., and T. Sasikala. "Efficient energy optimization in mobile ad hoc network (MANET) using better-quality AODV protocol." Cluster Computing (2018): 1-7.

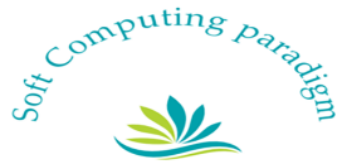


Journal of Soft Computing Paradigm (JSCP) (2019)

Vol.01/ No. 01

Pages: $1-13$

http://irojournals.com/jscp/

DOI: https://doi.org/10.36548/jscp.2019.1.001

[14]Bisen, Dhananjay, and Sanjeev Sharma. "Fuzzy based detection of malicious activity for security assessment of MANET." National Academy science letters 41, no. 1 (2018): 23-28.

[15]Lanjewar, Priyanka D., and V. M. Thakare. "Performance and Optimization of MANET Routing Protocols." International Journal of Computer Applications 975 (2015): 8887.

[16] https://www.geeksforgeeks.org/introduction-of-mobile-ad-hoc-network-manet/

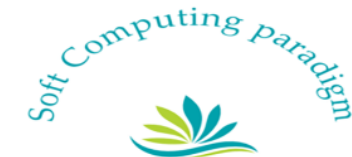

